\title{
Reliability of diffusion tensor tractography of facial nerve in cerebello-pontine angle tumours
}

\author{
Tomasz Szmuda ${ }^{1}$, Paweł Słoniewski ${ }^{1}$, Shan Ali ${ }^{2}$, Pedro M. Gonçalves Pereira ${ }^{3}$, Mateusz Pacholski ${ }^{2}$, \\ Fanar Timemy ${ }^{2}$, Agnieszka Sabisz ${ }^{4}$, Edyta Szurowska $^{4}$, Sara Kierońska ${ }^{1}$ \\ ${ }^{1}$ Neurosurgery Department, Medical University of Gdansk, Poland \\ ${ }^{2}$ Students' Scientific Circle of Neurology and Neurosurgery, Medical University of Gdansk, Poland \\ ${ }^{3}$ Algarve Biomedical Centre, University of Algarve, Faro, Portugal \\ ${ }^{4}$ Radiology Department, Medical University of Gdansk, Poland
}

\section{ABSTRACT}

Aim of the study. This study aimed to verify the accuracy of preoperative visualisation of the facial nerve (FN) by magnetic resonance-based (MR) diffusion tensor imaging-fibre tracking (DTI-FT) with neuronavigation system integration in patients with cerebello-pontine angle (CPA) tumours.

Clinical rationale for the study. Complete excision with preservation of the FN remains the critical goal of today's vestibular schwannoma (VS) surgery. DTI-FT of the FN with neuronavigation is yet to be fully evaluated, and could make surgery safer.

Materials and methods. This was a prospective cohort study in which 38 consecutive patients with a CPA tumour (32 VSs, five meningiomas and one epidermoid cyst) were operated on via the retrosigmoid route from 2013 to 2019. The course of the FN was simulated before surgery using StealthViz and the images were transferred to the Medtronic S7 neuronavigation system. The FN location reconstructed by DTI-FT was verified during the surgery.

Results. MR acquisition was inappropriate in three patients (7.9\%). DTI-FT correctly predicted the course of the FN in 31 of the 38 patients; the discordance rate was $18.4 \%$. The accuracy of DTI-FT was $81.6 \%(95 \% \mathrm{Cl}: 65.67-92.26)$, sensitivity $88.57 \%(95 \% \mathrm{Cl}$ : 73.26-96.80) and positive predictive value was $91.18 \%(95 \% \mathrm{Cl}: 90.17-92.09)$. The reliability of the neuronavigation-integrated visualisation of the FN did not depend on the tumour size $(p=0.85)$, but the method was more accurate when the nerve was compact in shape ( $p=0.03$, area under curve (AUC) $0.87,95 \% \mathrm{Cl}: 0.60-1.00$ ) and in females ( $p=0.04, \mathrm{AUC} 0.78,95 \% \mathrm{Cl}: 0.56-1.00$ ). Following surgery, $86.5 \%$ of the patients presented with useful facial function (House-Brackmann grades I-III). Correct simulation of the FN did not prevent postoperative facial palsy $(p=0.35)$.

Conclusions. The accuracy of DTI-FT of the FN integrated with neuronavigation remains unsatisfactory. This method does not provide any clinical benefit over non-integrated DTI-FT in terms of nerve function preservation.

Clinical implications. Due to the low reliability of the predictions, further technical advances in predicting the course of the FN are awaited by clinicians. However, DTI-FT images in the operating theatre would make tumour excision more comfortable for the surgeon.

Key words: cerebello-pontine angle, facial nerve, magnetic resonance imaging, diffusion tensor imaging, vestibular schwannoma (Neurol Neurochir Pol 2020; 54 (1): 73-82)

Address for correspondence: Tomasz Szmuda, Neurosurgery Department, Medical University of Gdansk, Dębinki 7 Str., 80-952 Gdansk, Poland, e-mail: tszmuda@gumed.edu.pl 


\section{Introduction}

Vestibular schwannomas (VSs), meningiomas and epidermoid cysts account for $80 \%$ of the tumours found within the cerebello-pontine angle (CPA) of the posterior fossa [1]. Beyond the excision of these tumours, facial nerve (FN) preservation is of vital importance, especially with VSs [2-6]. Intraoperative nerve injury not only affects a patient's quality of life, but is also a major cause of morbidity following the excision of a VS $[3,5]$. To date, the FN's course remains unpredictable [6]. In addition, nerve flattening or compression by a growing tumour limits the reliable visual identification of the FN during the surgery [3]. Due to these variables, safe surgery of VSs remains a challenge. Direct intraoperative electrophysiological monitoring has been used for early FN identification, but this technique remains inadequate $[3,7,8]$. For this reason, imaging techniques-based on conventional magnetic resonance (MR) cisternography [9] - have been developed that preoperatively simulate the position of the FN around the CPA tumour.

One recent example is diffusion tensor imaging-fibre tracking (DTI-FT) which is a non-invasive MR-based imaging modality. Since Taoka et al. first applied this technique to patients with VS, DTI-FT has emerged as a powerful technique in reconstructing the $\mathrm{FN}[3,4,9-11]$.

Despite the variable acquisition techniques, software and tracking methods, the predictive reliability of the FN's position in VS patients has reached $90.6 \%$. Unfortunately, this is still not complete enough for neurological surgery [4]. Furthermore, the authors of a systematic review on DTI-FT of FN concluded that the integration of preoperative planning with neuronavigation is still to be evaluated [4]. To date, only one 18-patient study has assessed the effect of DTI-FT-integrated neuronavigation [6]. In our study, we evaluated the clinical benefit of preserving the $\mathrm{FN}$ offered by this technique.

\section{Materials and methods}

\section{Study population}

Patients with a primary CPA tumour were prospectively enrolled into this study from April 2013 to August 2019 at the Neurosurgery Department of the Medical University of Gdańsk in Poland. The routine local protocol for patients with a suspected VS, meningioma or epidermoid cyst, covered the acquisition of the CPA tumours-adjusted MR protocol prior to surgery. Meningiomas objectively not affecting the course of the FN were excluded from analysis.

The cohort consisted of 38 patients, comprising 19 males and 19 females; the mean and median ages were 54.4 and 57.5 years, respectively. The most common histopathology was VS ( $\mathrm{n}=32,84.2 \%)$, followed by meningioma $(\mathrm{n}=5,13.2 \%)$. There was one epidermoid cyst (2.6\%) in our series. The mean tumour diameter was $29.6 \mathrm{~mm}$ ( $\mathrm{SD} \pm 12.7$, min-max 9-70), and $28.8 \mathrm{~mm}$ specifically for VS (SD \pm 10.9 ; min-max 9-48).
Of the VSs, three (9.4\%) were graded Koos I, 12 (37.5\%) Koos II, 12 (37.5\%) Koos III, and five (15.6\%) Koos IV [12]. All 32 patients with a VS presented with sensorineural hearing loss, but only one had facial palsy at admission.

All the tumours were approached via retrosigmoid craniectomy; other approaches such as a presigmoid approach were not considered. In order to avoid air embolisms, the patient was positioned prone and the extent of the osteotomy was tailored to the particular tumour size. Following a Cshaped dural incision, the cisterns were opened. The manner of the tumour excision depended on the histopathology. In meningiomas, the tumour was first detached from the dura by an electric cautery and then removed in pieces. In VSs, the internal acoustic meatus was opened by a high-speed coarse and diamond drills following initial debulking. Afterwards, the intracanalicular portion of the tumour was gently removed. The FN was identified with direct mono- or bipolar stimulation by applying pulsed constant-current stimulation (ISIS IOM, Inomed, Emmendingen, Germany; NeuroExplorer software v. 5.1). The nerve, cerebellum and pons were dissected, and then the tumour was excised. An ultrasonic aspiration knife facilitated the excision of most tumours. Facial nerve function was evaluated approximately two weeks after the surgery according to the House-Brackmann (HB) classification [13]. Facial palsy was recognised if the HB grade was II to VI.

Informed consent was obtained from all patients and the protocol of this study was approved by the local Ethics Committee. We adhered to the STROBE Statement, a checklist of items for observational studies [14].

\section{Imaging acquisition}

MR scans were acquired on a 1.5 tesla Siemens Magnetom Aera 1.5T (Erlangen, Germany) with a 20-channel head coil. The imaging protocol for CPA tumour included: $\mathrm{T} 1$, T2-weighted, constructive interference in steady state (CISS) and post-gadolinium T1-weighted (the last sequence served as a reference for neuronavigation). Diffusion-weighted imaging (DWI) was obtained by readout segmentation of long variable echo-trains high-definition protocol (predefined by the manufacturer, syngo RESOLVE ${ }^{\mathrm{TM}}$ ). The number of diffusion-encoding gradient directions was 20 , the $b$-factor was $0,1,000 \mathrm{~s} / \mathrm{mm}^{2}$ (see Table 2 for MR acquisition details). Except for bone masking, we did not perform any additional digital transformations such as motion or edgy corrections. The diffusion tensor of a single voxel was calculated using multivariate linear fitting. In that way, each voxel owned three eigenvectors, and the voxel associated with the largest eigenvalue denoted the ultimate fibre direction.

\section{Data processing}

The entire MR exam was imported to StealthViz software with a StealthDTI ${ }^{\mathrm{TM}}$ module (Medtronic Inc., Minneapolis, MN, USA), and later fused and manually verified. Based on the DWI sequence, the particular DTI derivate images were 
Table 1. Patient characteristics, outcomes and facial nerve fibre tracking

\begin{tabular}{|c|c|c|c|c|c|c|c|c|c|c|c|c|c|}
\hline$\stackrel{0}{z}$ & 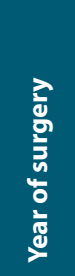 & $\stackrel{9}{8}$ & ณ̊ & 음 & $\frac{\text { 흥 }}{\frac{\circ}{\circ}}$ & 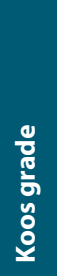 & 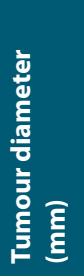 & 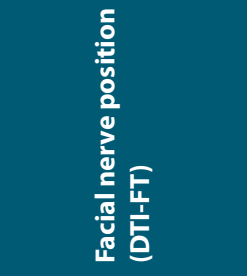 & 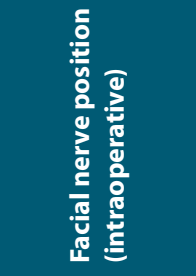 & 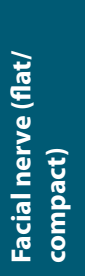 & 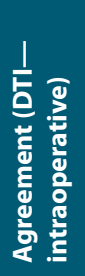 & 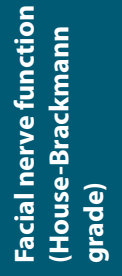 & 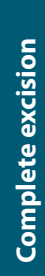 \\
\hline 1 & 2013 & 38 & $\mathrm{~F}$ & $\mathrm{R}$ & VS & 1 & 9 & anterior & anterior & C & 1 & 1 & 1 \\
\hline 2 & 2013 & 30 & $\mathrm{M}$ & $\mathrm{R}$ & VS & 2 & 21 & antero-superior & antero-inferior & Flat & 0 & 2 & 1 \\
\hline 3 & 2013 & 57 & M & $\mathrm{R}$ & VS & 2 & 25 & antero-superior & antero-superior & Flat & 1 & 3 & 1 \\
\hline 4 & 2013 & 58 & $M$ & $\mathrm{R}$ & $\begin{array}{l}\text { Epider- } \\
\text { moid cyst }\end{array}$ & NA & 70 & anterior & anterior & C & 1 & 1 & 0 \\
\hline 5 & 2014 & 69 & $\mathrm{~F}$ & $\mathrm{~L}$ & VS & 3 & 22 & antero-superior & antero-superior & C & 1 & 4 & 1 \\
\hline 6 & 2014 & 51 & $\mathrm{~F}$ & $\mathrm{R}$ & VS & 2 & 27 & anterior & anterior & $\mathrm{C}$ & 1 & 1 & 1 \\
\hline 7 & 2014 & 65 & $M$ & $\mathrm{R}$ & MG & NA & 19 & wrong MR acquisition & antero-medial & C & 0 & 1 & 1 \\
\hline 8 & 2014 & 43 & $M$ & $\mathrm{~L}$ & VS & 4 & 45 & penetrating & penetrating & Flat & 1 & 1 & 0 \\
\hline 9 & 2014 & 70 & M & $\mathrm{L}$ & VS & 4 & 45 & superior & superior & Flat & 1 & 1 & 0 \\
\hline 10 & 2014 & 62 & $\mathrm{M}$ & $\mathrm{R}$ & VS & 3 & 38 & superior & antero-superior & UNK & 0 & death & 0 \\
\hline 11 & 2014 & 37 & M & $\mathrm{R}$ & VS & 1 & 19 & superior & superior & Flat & 1 & 2 & 1 \\
\hline 12 & 2014 & 60 & $\mathrm{~F}$ & $\mathrm{~L}$ & VS & 1 & 10 & antero-superior & antero-superior & C & 1 & 1 & 1 \\
\hline 13 & 2015 & 61 & $M$ & $\mathrm{~L}$ & VS & 3 & 39 & posterior & anterior & Flat & 0 & 6 & 1 \\
\hline 14 & 2015 & 35 & $\mathrm{~F}$ & $\mathrm{R}$ & VS & 4 & 48 & inferior & inferior & UNK & 1 & 6 & 1 \\
\hline 15 & 2015 & 71 & $M$ & $\mathrm{~L}$ & VS & 3 & 32 & antero-inferior & antero-inferior & C & 1 & 1 & 0 \\
\hline 16 & 2015 & 81 & $M$ & $\mathrm{~L}$ & VS & 3 & 23 & UNK & antero-superior & Flat & 0 & 1 & 1 \\
\hline 17 & 2015 & 71 & $\mathrm{~F}$ & $\mathrm{R}$ & VS & 4 & 38 & postero-superior & postero-superior & Flat & 1 & 3 & 0 \\
\hline 18 & 2016 & 54 & $\mathrm{~F}$ & $\mathrm{~L}$ & VS & 3 & 27 & antero-inferior & antero-inferior & C & 1 & 1 & 1 \\
\hline 19 & 2016 & 57 & $M$ & $\mathrm{R}$ & VS & 2 & 10 & antero-superior & antero-superior & C & 1 & 1 & 1 \\
\hline 20 & 2016 & 25 & $M$ & $\mathrm{R}$ & VS & 2 & 36 & antero-superior & antero-superior & C & 1 & 1 & 0 \\
\hline 21 & 2017 & 49 & $M$ & $\mathrm{R}$ & VS & 2 & 29 & wrong MR acquisition & antero-superior & C & 0 & 2 & 1 \\
\hline 22 & 2017 & 56 & $\mathrm{~F}$ & $\mathrm{R}$ & MG & NA & 43 & anterior & anterior & C & 1 & 1 & 1 \\
\hline 23 & 2017 & 50 & $\mathrm{~F}$ & $\mathrm{~L}$ & VS & 4 & 43 & postero-inferior & postero-inferior & Flat & 1 & 1 & 0 \\
\hline 24 & 2017 & 43 & $\mathrm{~F}$ & $\mathrm{~L}$ & VS & 2 & 16 & wrong MR acquisition & anterior & C & 0 & 1 & 1 \\
\hline 25 & 2017 & 35 & $\mathrm{~F}$ & $\mathrm{~L}$ & VS & 3 & 35 & antero-superior & antero-superior & C & 1 & 4 & 1 \\
\hline 26 & 2017 & 32 & $\mathrm{~F}$ & $\mathrm{R}$ & VS & 3 & 41 & antero-superior & antero-superior & Flat & 1 & 5 & 1 \\
\hline 27 & 2018 & 63 & $\mathrm{~F}$ & $\mathrm{~L}$ & VS & 2 & 37 & antero-superior & antero-superior & C & 1 & 2 & 1 \\
\hline 28 & 2018 & 48 & $M$ & $\mathrm{R}$ & VS & 2 & 33 & superior & superior & C & 1 & 1 & 1 \\
\hline 29 & 2018 & 72 & $\mathrm{~F}$ & $\mathrm{~L}$ & VS & 2 & 17 & antero-superior & antero-superior & C & 1 & 1 & 1 \\
\hline 30 & 2018 & 50 & $\mathrm{~F}$ & $\mathrm{~L}$ & VS & 3 & 30 & antero-superior & antero-superior & C & 1 & 1 & 0 \\
\hline 31 & 2018 & 58 & $\mathrm{~F}$ & $\mathrm{~L}$ & VS & 2 & 31 & antero-superior & antero-superior & C & 1 & 1 & 1 \\
\hline 32 & 2018 & 59 & $M$ & $\mathrm{R}$ & VS & 3 & 19 & superior & superior & C & 1 & 1 & 1 \\
\hline 33 & 2018 & 62 & $M$ & $\mathrm{~L}$ & VS & 3 & 36 & antero-superior & antero-superior & Flat & 1 & 2 & 0 \\
\hline 34 & 2018 & 44 & $\mathrm{~F}$ & $\mathrm{R}$ & MG & NA & 12 & anterior & anterior & C & 1 & 1 & 1 \\
\hline 35 & 2019 & 62 & $\mathrm{~F}$ & $\mathrm{R}$ & VS & 3 & 22 & antero-superior & antero-superior & C & 1 & 3 & 1 \\
\hline 36 & 2019 & 67 & $M$ & $\mathrm{~L}$ & MG & NA & 23 & anterior & anterior & C & 1 & 1 & 1 \\
\hline 37 & 2019 & 61 & $M$ & $\mathrm{R}$ & VS & 2 & 18 & antero-superior & antero-superior & C & 1 & 2 & 1 \\
\hline 38 & 2019 & 60 & $\mathrm{~F}$ & $\mathrm{~L}$ & MG & NA & 35 & anterior & anterior & C & 1 & 1 & 1 \\
\hline
\end{tabular}


Table 2. Main parameters of magnetic resonance sequences

\begin{tabular}{|c|c|c|c|c|c|c|c|c|c|c|}
\hline $\begin{array}{c}\text { Sequence } \\
\text { type }\end{array}$ & Plane & $\begin{array}{l}\text { Name of } \\
\text { specific } \\
\text { techni- } \\
\text { que }\end{array}$ & $\begin{array}{l}\text { Field-of- } \\
\text {-view [mm } \\
x \text { mm] }\end{array}$ & $\begin{array}{c}\text { Voxel size } \\
\text { [mm x mm x } \\
\mathrm{mm}]\end{array}$ & $\begin{array}{l}\text { Gap } \\
{[\%]}\end{array}$ & $\begin{array}{l}\text { Num- } \\
\text { ber of } \\
\text { slices }\end{array}$ & $\begin{array}{l}\text { Repeti- } \\
\text { tion Time } \\
\text { [ms] }\end{array}$ & $\begin{array}{l}\text { Echo } \\
\text { Time } \\
\text { [ms] }\end{array}$ & $\begin{array}{l}\text { Time of } \\
\text { Inversion } \\
\text { [ms] }\end{array}$ & $\begin{array}{c}\text { Signal } \\
\text { Averaging }\end{array}$ \\
\hline T2-weighted & sagittal & blade & $250 \times 250$ & $0.7 \times 0.7 \times 4.0$ & 20 & 30 & 4,430 & 146 & - & 1 \\
\hline T2-weighted & coronal & TSE & $250 \times 250$ & $0.5 \times 0.5 \times 4.0$ & 20 & 28 & 5,180 & 109 & - & 2 \\
\hline $\begin{array}{l}\text { T2-weighted } \\
\text { FLAIR }\end{array}$ & transverse & TIR & $260 \times 187$ & $0.4 \times 0.4 \times 4.0$ & 20 & 30 & 9,000 & 106 & 2,500 & 1 \\
\hline T2-weighted & transverse & CISS & $220 \times 165$ & $0.4 \times 0.4 \times 0.7$ & 20 & 88 & 5.66 & 2.83 & - & 1 \\
\hline DTI & transverse & RESOLVE & $230 \times 230$ & $1.5 \times 1.5 \times 1.5$ & 0 & 30 & 4,870 & $\begin{array}{c}74 \text { and } \\
111\end{array}$ & - & 2 \\
\hline T2-weighted & transverse & TSE & $250 \times 187$ & $0.6 \times 0.6 \times 2.0$ & 0 & 80 & 15,960 & 89 & - & 1 \\
\hline T1-weighted & transverse & MPRAGE & $260 \times 260$ & $1.0 \times 1.0 \times 1.0$ & 50 & 176 & 1,730 & 3.27 & 800 & 1 \\
\hline
\end{tabular}

$\mathrm{mm}$ - millimetres; TSE — turbo spin echo; TIR — triple inversion recovery; CISS — constructive interference in steady state; DTI — diffusion tensor imaging

automatically calculated, including directionally encoded colours, apparent diffusion coefficient, fractional anisotropy (FA) and mean diffusivity. In order to track the nerve, a single region-of-interest was set in the entire internal acoustic meatus. The FA start value was 0.2 and the angle threshold was $>60^{\circ}$, although these values were balanced individually during post-processing. The thicker bundle starting from the meatus and rounding the tumour was visualised and recorded as a predicted location of the FN. To define the course of the $\mathrm{FN}$, the angle of view was adjusted to the perspective proposed by Sampath et al. [15].

Accordingly, the FN location in relation to the tumour was categorised as: anterior, antero-superior, superior, posterior or inferior. Multiplanar reconstructions as well as three-dimensional modelling enabled assessment of the structures in various planes as well as with the retrosigmoid approach. Both the tumour volume and the predicted course of the FN were segmented, and exported, together with the reference exam (contrast-enhanced T1-weighted sequence) to the neuronavigation system (StealthStation S7, Medtronic Inc., Minneapolis, MN, USA). The surgical microscope (Pentero 800, Carl Zeiss AG, Oberkochen, Germany) was fully integrated with the navigation system, with a display that was updated in real time with the microscope's movement. Both the surgeries and the neuronavigation display were recorded simultaneously; these videos were used for post-hoc analysis between the predicted course of the FN and the actual course found during the surgery. Unlike other studies [7, 16], the surgeon was not blinded to the results of preoperative DTI-FT. The segmented tumour and the FN (prepared by the first author) were seen by the surgeon (the senior author) in the navigation display and/or in the injected picture in the surgical microscope. See the supplementary video showing how the FN was tracked.

\section{Outcome measures}

The single outcome measure was the prediction accuracy of the FN's path made with DTI-FT integrated with a neuronavigation system. Intraoperative monitoring and/or visual recognition of the FN confirmed the actual course of the FN. The sensitivity and specificity of DTI-FT was calculated. We also aimed to associate the clinical and radiological factors with agreement between the predicted and the actual position of the nerve.

\section{Statistical methods}

Continuous variables were described by the mean, standard deviation and range, and were compared using the Mann-Whitney U test. Chi-square and two-tailed Fisher's exact test was used for the analysis of categorical data.

We used software for particular statistical analyses: Statistica v. 13.1 (StatSoft Co, Tulsa, OK, USA) and Prism v. 6.07 (GraphPad Software, La Jolla, CA, USA). A p value below 0.05 was deemed significant. Receiver operating characteristic curves for significant variables were created and the

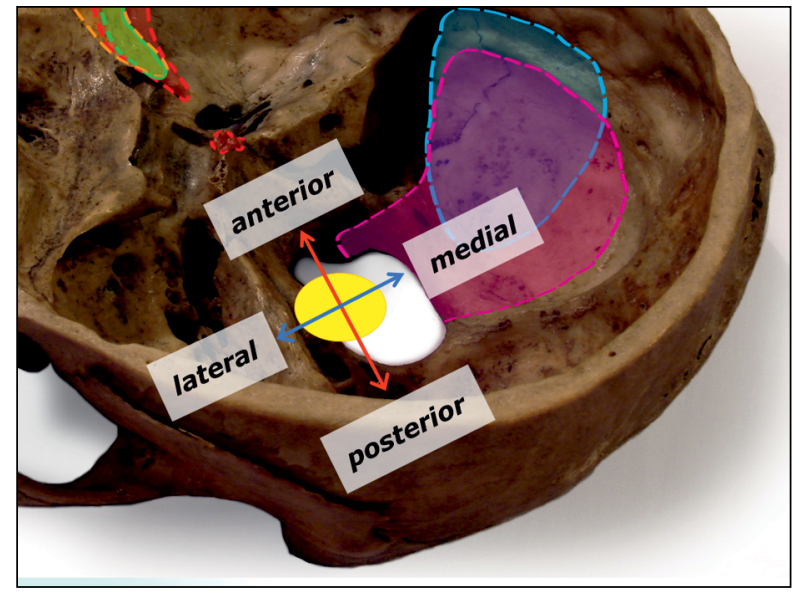

Figure 1. This illustration of the left-sided vestibular schwannoma demonstrates the possible positions of the facial nerve according to the Sampath classification [15]. Typical retrosigmoid craniectomy is marked in blue, and the extended craniectomy to the foramen magnum that was used for large tumours is marked in violet. 

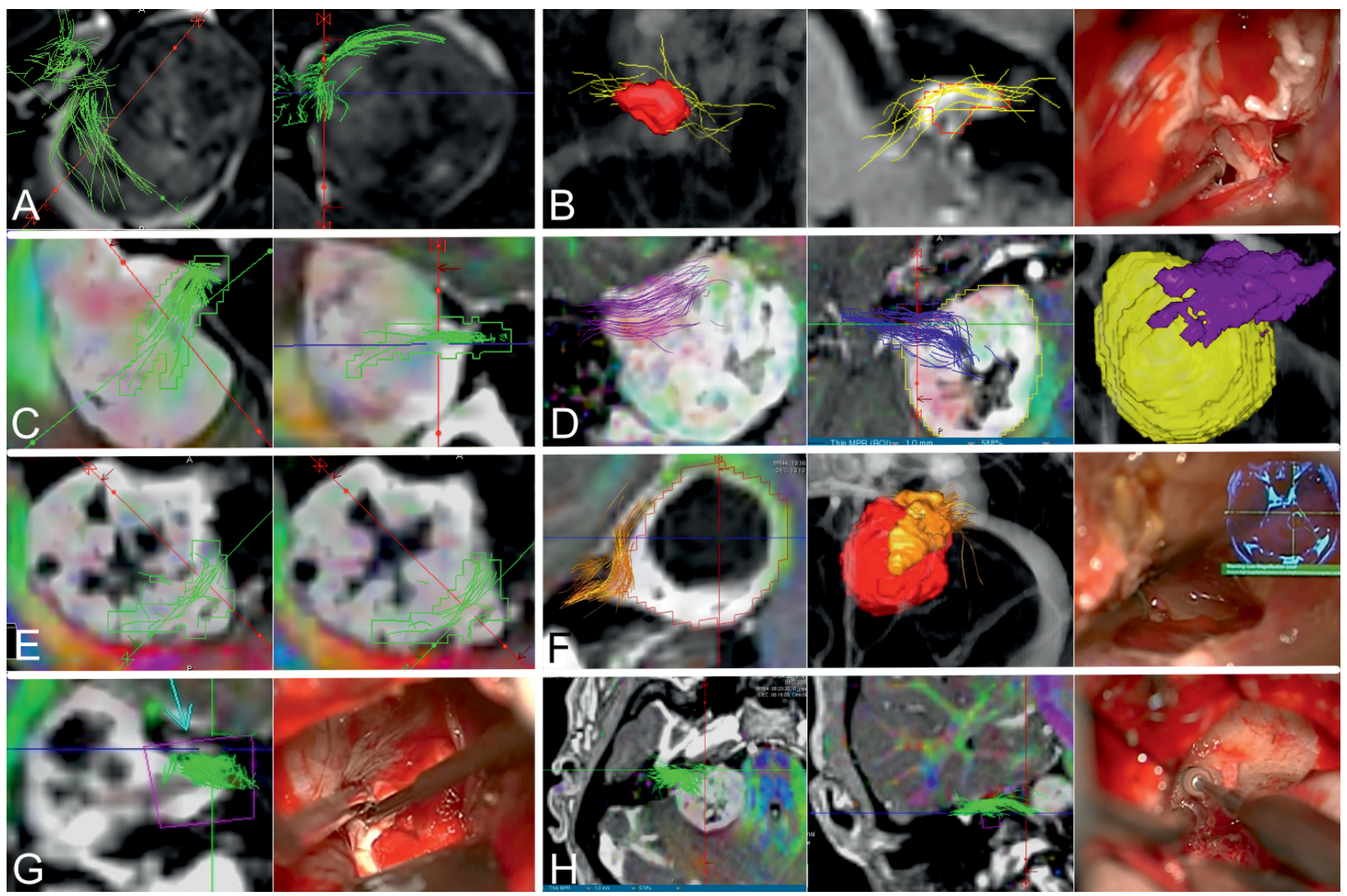

Figure 2. Ilustration of facial nerve (FN) courses (colour bundles) tracked by diffusion tensor imaging. All presented cases are vestibular schwannomas.

A. Case 17: Postero-superior, axial view, coronal view.

B. Case 12: Antero-superior, three-dimensional model, axial view and intraoperative snapshot.

C. Case 32: Superior, axial views.

D. Case 9: Superior, axial views and three-dimensional model.

E. Case 33: Antero-superior, axial views.

F. Case 3: Antero-superior, axial view, three-dimensional model and intraoperative snapshot with injected quarter-screen picture of real-time neuronavigation.

G. Case 5: Antero-superior, sagittal view and intraoperative snapshot.

H. Case 6: Anterior, axial, coronal view and intraoperative snapshot.

area under the curves (AUC) were compared in MedCalc v. 11.4 (MedCalc Software; Mariakerke, Belgium).

\section{Results}

\section{Surgical outcome}

The postoperative course, as well as the final clinical outcome, were uneventful in all patients, except for one mortality due to an idiopathic vast pontine oedema. Surgery-related facial palsy (HB grade II-VI) was recognised in 14 patients $(14 / 37 ; 36.8 \%)$. No or minor facial palsy (known as 'useful facial function, HB grades I-III) was present in 32 patients (32/37; $86.5 \%)$. Since all surgeries were done in the prone position, we did not encounter any air embolism. There was no sinus or vertebral artery injury during the craniotomy. Other intraoperative complications included a local haematoma requiring surgical revision in one patient. Postoperative cerebrospinal fluid leakage was encountered in $4 / 37$ patients (10.8\%) and was treated successfully by external lumbar drainage, although the hospital stay was prolonged in these patients.

\section{Facial nerve prediction}

In the 35 patients where DTI was comprehensive, the correct course of the FN was seen in 24 cases $(24 / 35 ; 68.6 \%)$. In 10 cases $(10 / 35 ; 28.6 \%)$ the nerve's path was highly predictive as the main fibre bundle determined the FN's position. The FN was not visualised in one individual $(1 / 35 ; 2.9 \%)$ with a cystic VS (23 $\mathrm{mm}$ in diameter).

In our study, DTI-FT correctly predicted the course of the FN in 31 out of 38 patients (81.6\%). However, the prediction accuracy jumped to 31 out of 35 patients $(88.6 \%)$ when we only included patients in whom DTI-FT was performed properly. Regarding the subset of VSs, DTI-FT was correct in simulating the FN's position in 26 out of 30 patients (86.7\%). 
Among VSs, the antero-superior (17/38, 44.7\%) and anterior $(9 / 38,23.7 \%)$ were the most common positions of the FN around the tumour according to the Sampath classification [15]. DTI-FT was more accurate when the nerve was not flat $(p=0.03)$ and in females $(p=0.04)$. The identification rate did not differ significantly $(p=0.43)$ in regard to the FN's position. The reliability of DTI-FT for the FN did not depend on other analysed factors such as tumour size $(\mathrm{p}=0.85)$. Receiver operating characteristic curves for the FN shape and sex were subsequently compared; AUC was 0.87 (95\% CI: $0.60-1.00)$ and 0.78 (95\% CI: 0.56-1.00), respectively. We found that the shape of the FN was the dominant component influencing the accuracy of DTI-FT, as there was a larger AUC for a compactly shaped FN. However, the comparison of AUCs was not significant $(\mathrm{p}=0.63)$.

The accuracy of the preoperative visualisation of the FN did not prevent facial palsy ( $\mathrm{p}=0.35)$; the AUC for the House-Brackmann grade was 0.69 (95\% CI: 0.34-1.00).

The calculated accuracy DTI-FT was $81.58 \%$ (95\% CI: 65.67 to 92.26 ). The comprehensive predictive capability of DTI-FT was additionally evaluated by tools for a diagnostic test evaluation. These values were respectively: sensitivity $88.57 \%$ (95\% CI: 73.26 to 96.80 ), specificity $0 \%$ (95\% CI: 0 to 70.76 ) and positive predictive value of $91.18 \%$ (95\% CI: 90.17 to 92.09 ).

\section{Discussion}

We found that the FN's path can be predicted correctly with preoperative DTI-FT visualisations in four-fifths of patients $(81.6 \%)$ with a CPA tumour; but this is far from the long-desired goal in neurosurgery. Moreover, the integration of surgical planning with the neuronavigation system did not increase the prediction rate when compared to previous studies solely relying on non-integrated preoperative DTI-FT simulations $[3,4]$. Today, there is growing interest in the reliable identification of the FN in CPA angle tumours, so further advances are necessary $[3,4]$. Our findings are novel because the integration of preoperative planning with neuronavigation is only just beginning to be explored in neurological surgery.

Since DTI-FT of cranial nerves first came to the attention of the neurosurgical community, fewer than 30 research groups have investigated its benefits in the surgery of CPA [11]. Yet during these 13 years, the concordance between the radiological reconstruction of the FN and intraoperative findings has substantially improved. Taoka et al. was the first to report the use of DTI-FT in VSs, but attained a low agreement that barely exceeded 60\% [9]. Our initial results were also not promising [17]. However, three later studies overcame some technical constraints and demonstrated significant correlations between preoperative imaging and the FN's actual position [18-20]. Notably, these initial reports included only a small number of patients. Larger studies revealed that DTI-FT-based nerve predictions were not always correct $[6,21,22]$. In larger series, the DTI-FT-based reconstruction of the FN yielded 91\% reliable predictions [23]. Researchers have tried DTI-FT augmented with high-definition ultrasonography, although their initially promising results have not been followed up by other physicians [8]. We feel that these varying results are due to the highly variable methodology, MR field strengths, number of diffusion directions, software and tracking techniques used in each study.

Four systematic reviews have summarised the findings of DTI-FT in VSs $[3,4,11,24]$. Two of these reviews pooled all the published cases into one artificial single subset by using the individual patient data PRISMA methodology (or equivalent) $[3,4]$. In that way, Baro et al. attempted to conclude from a highly unselected cohort of 223 individuals [3]. The overall incongruence between DTI-FT reconstruction and intraoperative nerve position was $13.3 \%$ (95\% CI: 5.7-27.8), and the overall accuracy was $85.5 \%$ [3]. We did not surpass that figure, given that only $81.6 \%$ of our predictions were true. Baro et al. listed the main factors responsible for the incongruence: structural alterations of the FN, cystic VSs, technical problems related to the acquisition, post-processing, and anatomical alterations in large VSs [3]. The second systematic review, by Savardekar et al., pooled 234 original VSs and obtained a high, $90.6 \%$, accuracy of DTI-FT of the FN [4]. Although they did not analyse the factors related to discordance, integration of the neuronavigation system with the prepared DTI-FT images needs verification in terms of decreasing the neurological complication rate [4].

Therefore, our study met the demand for preoperative visualisation of the FN by DTI-FT and a tumour that is integrated with the neuronavigation system [4]. Li et al. first integrated neuronavigation with the preoperative DTI-FT images with correct predictions in 17 out of 18 patients [6]. The authors suggested that integration with neuronavigation offered a wide range of applications, such as removing unnecessary worry when debulking in the region where the FN is not indicated by the navigation [6]. We feel that stereotactic localisation of the FN could be clinically useful to the neurosurgeon, but only with complete or nearly complete reliability. Failing that, a surgeon could mistakenly injure a nerve, or look for a nerve where it is not present. Our study proved that this imaging technique currently requires more development.

All DTI-FT software settings, including the thresholds of various values, region-of-interests and cropping fibres, are highly operator-dependent. This creates a situation where two DTI-FT operators can produce two different tracing results [6]. Therefore, the value of literature reviews mentioned earlier is debatable because studies differ substantially in their imaging methodology. In our study, we modified the FA thresholds and other parameters to obtain a more reliable FN visualisation based on previous research [25]. However, we deliberately set only one region-of-interest in the entire internal auditory canal since we found that setting an additional region-of-interest at the pons was ineffective. Peculiar diffusion around the CPA 
Table 3. Factors associated with accuracy of diffusion tensor tractography-fibre tracking of facial nerve

\begin{tabular}{|c|c|c|}
\hline Factor & Correct prediction & Incorrect prediction \\
\hline \multirow[t]{2}{*}{ Age $($ mean $\pm S D)$} & $54.0 \pm 12.7$ & $58.5 \pm 21.1$ \\
\hline & & \\
\hline \multirow[t]{2}{*}{ Sex ( $n, \%$ of females) } & $18 / 31,58.1 \%$ & $0 / 4,0 \%$ \\
\hline & & \\
\hline \multirow[t]{2}{*}{ Side ( $n, \%$ of left) } & $15 / 17,88.2 \%$ & $2 / 17,11.8 \%$ \\
\hline & & \\
\hline \multirow[t]{2}{*}{ Years from study initiation (mean $\pm S D$ ) } & $3.3 \pm 2.0$ & $1.3 \pm 1.0$ \\
\hline & & \\
\hline \multicolumn{3}{|l|}{ Tumour and facial nerve } \\
\hline \multicolumn{3}{|l|}{ Pathology } \\
\hline Vestibular schwannoma $(n, \%)$ & $26 / 30,86.7 \%$ & $4 / 30,100 \%$ \\
\hline \multirow[t]{2}{*}{ Meningioma $(n, \%)$} & $4 / 4,100 \%$ & $0 / 4,0 \%$ \\
\hline & & \\
\hline \multirow[t]{2}{*}{ Intracanalicular growth $(n, \%)$} & $23 / 27,85.2 \%$ & $4 / 27,14.8 \%$ \\
\hline & & \\
\hline \multirow[t]{2}{*}{ Diameter $($ mean $\pm S D)$} & $30.3 \pm 13.4$ & $30.2 \pm 9.6$ \\
\hline & & \\
\hline \multirow[t]{2}{*}{ Koos grade (mean $\pm S D$ ) } & $2.6 \pm 0.9$ & $2.8 \pm 0.5$ \\
\hline & & \\
\hline \multirow[t]{2}{*}{ Koos grade $\geq 3(n, \%)$} & $14 / 17,82.4 \%$ & $3 / 17,17.6 \%$ \\
\hline & & \\
\hline \multirow[t]{2}{*}{ Tumour diameter (mean $\pm S D)$} & $30.3 \pm 13.4$ & $30.3 \pm 9.6$ \\
\hline & & \\
\hline \multirow[t]{2}{*}{ Tumour diameter $\geq 3 \mathrm{~cm}(n, \%)$} & $17 / 19,89.5 \%$ & $2 / 19,10.5 \%$ \\
\hline & & \\
\hline \multirow[t]{2}{*}{ Compact shape of facial nerve $(n, \%)$} & $8 / 11,72.7 \%$ & $3 / 11,27.3 \%$ \\
\hline & & \\
\hline \multicolumn{3}{|l|}{ Facial nerve position (Sampath $[15], n, \%$ ) } \\
\hline \multirow[t]{2}{*}{ Antero-superior } & $14 / 16,87.5 \%$ & $2 / 14,12.5 \%$ \\
\hline & & \\
\hline \multirow[t]{2}{*}{ Anterior } & $7 / 8,87.5 \%$ & $1 / 8,12.5 \%$ \\
\hline & & \\
\hline \multirow[t]{2}{*}{ Superior } & $4 / 4,100 \%$ & $0 / 4,0 \%$ \\
\hline & & \\
\hline \multirow[t]{2}{*}{ Other } & $6 / 7,85.7 \%$ & $1 / 7,14.3 \%$ \\
\hline & & \\
\hline \multicolumn{3}{|l|}{ Outcome } \\
\hline \multirow[t]{2}{*}{ HB grade $\geq 2(n, \%)$} & $11 / 13,84.6 \%$ & $2 / 13,15.4 \%$ \\
\hline & & \\
\hline \multirow[t]{2}{*}{$\mathrm{HB}$ grade (mean $\pm S D$ ) } & $1.8 \pm 1.4$ & $3.0 \pm 2.6$ \\
\hline & & \\
\hline \multirow[t]{2}{*}{ Complete excision ( $n, \%)$} & $18 / 21,85.7 \%$ & $3 / 21,14.3 \%$ \\
\hline & & \\
\hline
\end{tabular}

HB - House-Brackmann grade of facial palsy; SD - standard deviation 


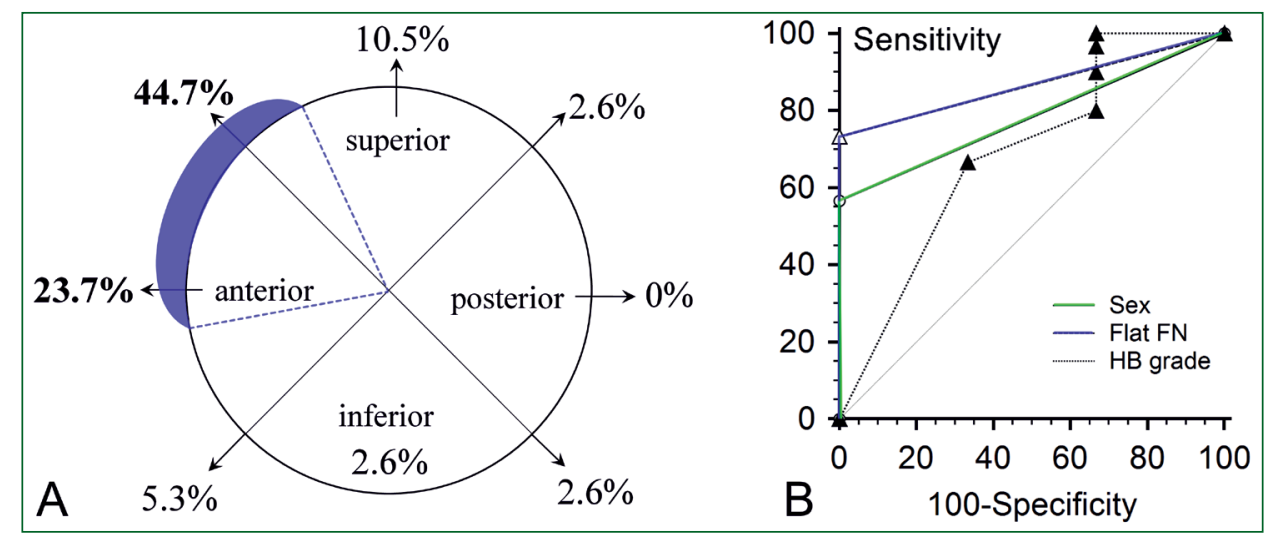

Figure 3. A. Illustration showing frequency of locations of facial nerve (according to classification proposed by Sampath et al. [15]). B. Receiver operating characteristic curves of factors influencing prediction rate for diffusion tensor imaging-fibre tracking (DTI-FT) of facial nerve (FN). House-Brackmann grading used to demonstrate that DTI-FT of FN had positive influence on neurological outcome, although not significant $\mathrm{FN}$ - facial nerve, HB grade - House-Brackmann grade of facial palsy

Table 4. Prediction of facial nerve location in relation to intraoperative findings

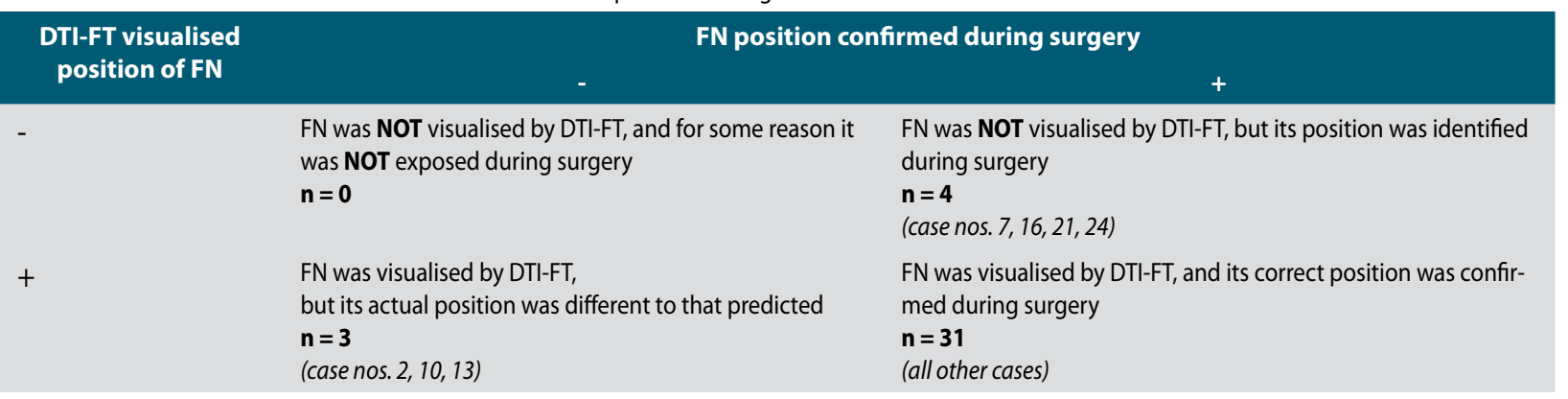

DTI-FT — diffusion tensor imaging-fibre tracking; $\mathrm{FN}$ - facial nerve

precludes drawing long fibres, hence we selected the main DTI bundle around the tumour which was passing through the internal auditory canal.

Even so, the low accuracy of our method may indicate that we performed DTI-FT ineffectively. FN reconstructions using probabilistic tractography were considered to solve the problem of many fibres which are densely packed around the VS. Unfortunately, the obtained accuracy was not satisfactory and reached only $81 \%$ [26]. A fully reliable method visualising the FN is still needed. Recalculation of a few co-registered MR sequences could be the solution. Initially, this did not increase the reliability of FN prediction, although DTI together with CISS sequence could somewhat differentiate the FN from the cochlear nerve $[23,27]$. Recently, Pereira et al. developed a promising concept involving fusing sequences: CISS, $\mathrm{T} 1$ and contrast-enhanced T1. This method takes advantage of the non-contrasting regularity of the FN as compared to the tumour [28]. In this preliminary report on five cases, the agreement between the fused CISS/T1 and intraoperative findings was always correct.

Therefore, we feel that determining the right course of the FN in CPA tumours is not just a matter of improving a single DTI sequence, but also of combining semi-automated and acquired sequences. Skilful fusion techniques have not reached the limits of their capabilities, and are promising in terms of forthcoming FN identification [28]. Perhaps in the future neural networks could help streamline imaging of the cranial nerves and overcome human limitations. Unless augmented by other technical advances, we assert that the development of an isolated DTI technique for FN tracking will not attain optimal accuracy.

We compared our complication rates with a recent study on 502 VSs [29]. In this study, approximately $25 \%$ had facial palsy (HB grades II-VI), whereas $36.8 \%$ of our patients had it. In terms of preserved useful facial functions (HB grades I-III), we encountered the same percentage: $86 \% .10 \%$ of our patients encountered cerebrospinal fluid leakage, which was similar to other series [29]. Complications, both neurological and general surgical, are the major concern in the modern approach to a VS. Over the last decade, we have witnessed the treatment paradigm of large VSs favouring subtotal resection, and subsequent stereotactic radiosurgery; this approach offers excellent functional outcomes while achieving a tumour control rate comparable to that after total resection [2].

Even with 38 patients in our study, we could have encountered some degree of a type II error. However, we emphasise 
that even if the study period was prolonged (and so the number of patients was increased) the FN prediction rate would not be affected significantly. We feel that the limitations of DTI-FT are in part associated with the practical application of the imaging technique. For example, it is not unusual to encounter a brain shift related to head misalignment, hardware micro-movements and cerebrospinal fluid release following partial tumour debulking. All these factors increase the inaccuracy of DTI-FT of the FN. Fortunately, future technical advances may address some of these variables e.g. making machinery more stable. An anatomical limitation also exists; the FN is usually tracked together with the vestibulocochlear nerve, and it is not always possible to distinguish them by DTI-FT.

\section{Conclusions}

The reliability of DTI-FT when predicting the FN's course remains unsatisfactory. Our study shows no benefit with DTI-FT neuronavigation integration over only preoperative DTI-FT visualisation. However, introducing surgical planning to the operating theatre can make excising the CPA tumour more comfortable for the surgeon. Combining more imaging modalities would increase the ability to predict the course of the FN, and reduce surgery-related complications.

\section{Abbreviations:}

AUC - area under the curve

CISS - constructive interference in steady state

$\mathrm{CPA}$ - cerebello-pontine angle

DTI - diffusion tensor imaging

DTI-FT - diffusion tensor imaging-fibre tracking

FA - fractional anisotropy

$\mathrm{FN}$ - facial nerve

HB - House-Brackmann

MRI - magnetic resonance imaging

VS - vestibular schwannoma

Funding: No funding was received for this research.

Conflict of Interest: All authors certify that they have no affiliation with or involvement in any organisation or entity with any financial interest (such as honoraria, educational grants, participation in speakers' bureaux, membership, employment, consultancies, stock ownership, or other equity interest, and expert testimony or patent-licensing arrangements), or non-financial interest (such as personal or professional relationships, affiliations, knowledge or beliefs) in the subject matter or materials discussed in this manuscript.

Ethical permission: For preparation of this article, ethical approval was obtained from the institution (IRB Committee number: NKEBN/98/2012, NKEBN/65/2019).

Informed consent: Informed consent for treatment and data processing was obtained from all individual participants included in this study.

\section{References}

1. Rinaldi V, Casale M, Bressi F, et al. Facial nerve outcome after vestibular schwannoma surgery: our experience. J Neurol Surg B Skull Base. 2012; 73(1): 21-27, doi: 10.1055/s-0032-1304559, indexed in Pubmed: 23372991.

2. Starnoni D, Daniel RT, Tuleasca C, et al. Systematic review and meta-analysis of the technique of subtotal resection and stereotactic radiosurgery for large vestibular schwannomas: a "nerve-centered" approach. Neurosurg Focus. 2018; 44(3): E4, doi: 10.3171/2017.12. FOCUS17669, indexed in Pubmed: 29490553.

3. Baro V, Landi A, Brigadoi S, et al. Preoperative Prediction of Facial Nerve in Patients with Vestibular Schwannomas: The Role of Diffusion Tensor Imaging-A Systematic Review. World Neurosurg. 2019; 125: 24-31, doi: 10.1016/j.wneu.2019.01.099, indexed in Pubmed: 30708084.

4. Savardekar AR, Patra DP, Thakur JD, et al. Preoperative diffusion tensor imaging-fiber tracking for facial nerve identification in vestibular schwannoma: a systematic review on its evolution and current status with a pooled data analysis of surgical concordance rates. Neurosurg Focus. 2018; 44(3): E5, doi: 10.3171/2017.12.FOCUS17672, indexed in Pubmed: 29490547.

5. Samala R, Borkar SA, Sharma R, et al. Effectiveness of preoperative facial nerve diffusion tensor imaging tractography for preservation of facial nerve function in surgery for large vestibular schwannomas: Results of a prospective randomized study. Neurol India. 2019; 67(1): 149-154, doi: 10.4103/0028-3886.253631, indexed in Pubmed: 30860114.

6. Li H, Wang L, Hao S, et al. Identification of the Facial Nerve in Relation to Vestibular Schwannoma Using Preoperative Diffusion Tensor Tractography and Intraoperative Tractography-Integrated Neuronavigation System. World Neurosurg. 2017; 107: 669-677, doi: 10.1016/j. wneu.2017.08.048, indexed in Pubmed: 28826862.

7. Borkar SA, Garg A, Mankotia DS, et al. Prediction of facial nerve position in large vestibular schwannomas using diffusion tensor imaging tractography and its intraoperative correlation. Neurol India. 2016; 64(5): 965-970, doi: 10.4103/0028-3886.190270, indexed in Pubmed: 27625239.

8. Simon NG, Cage T, Narvid J, et al. High-resolution ultrasonography and diffusion tensor tractography map normal nerve fascicles in relation to schwannoma tissue prior to resection. J Neurosurg. 2014; 120(5): 11131117, doi: 10.3171/2014.2.JNS131975, indexed in Pubmed: 24628610.

9. Taoka T, Hirabayashi H, Nakagawa H, et al. Displacement of the facial nerve course by vestibular schwannoma: preoperative visualization using diffusion tensor tractography. J Magn Reson Imaging. 2006; 24(5): 1005-1010, doi: 10.1002/jmri.20725, indexed in Pubmed: 17031835.

10. Jacquesson T, Frindel C, Kocevar G, et al. Overcoming Challenges of Cranial Nerve Tractography: A Targeted Review. Neurosurgery. 2019; 84(2): 313-325, doi: 10.1093/neuros/nyy229, indexed in Pubmed: 30010992.

11. Shapey J, Vos SB, Vercauteren T, et al. Clinical Applications for Diffusion MRI and Tractography of Cranial Nerves Within the Posterior Fossa: A Systematic Review. Front Neurosci. 2019; 13: 23, doi: 10.3389/ fnins.2019.00023, indexed in Pubmed: 30809109.

12. Koos WT, Day JD, Matula C, et al. Neurotopographic considerations in the microsurgical treatment of small acoustic neurinomas. J Neurosurg. 1998; 88(3): 506-512, doi: 10.3171/jns.1998.88.3.0506, indexed in Pubmed: 9488305. 
13. Sun MZ, Oh MC, Safaee M, et al. Neuroanatomical correlation of the House-Brackmann grading system in the microsurgical treatment of vestibular schwannoma. Neurosurg Focus. 2012; 33(3): E7, doi: 10.3171/2012.6.FOCUS12198, indexed in Pubmed: 22937858.

14. Elm Ev, Altman D, Egger M, et al. The Strengthening the Reporting of Observational Studies in Epidemiology (STROBE) statement: guidelines for reporting observational studies. Journal of Clinical Epidemiology. 2008; 61(4): 344-349, doi: 10.1016/j.jclinepi.2007.11.008.

15. Sampath $P$, Rini D, Long DM. Microanatomical variations in the cerebellopontine angle associated with vestibular schwannomas (acoustic neuromas): a retrospective study of 1006 consecutive cases. J Neurosurg. 2000; 92(1): 70-78, doi: 10.3171/jns.2000.92.1.0070, indexed in Pubmed: 10616085.

16. Churi ON, Gupta S, Misra BK. Correlation of Preoperative Cranial Nerve Diffusion Tensor Tractography with Intraoperative Findings in Surgery of Cerebellopontine Angle Tumors. World Neurosurg. 2019; 127: e509-e516, doi: 10.1016/j.wneu.2019.03.190, indexed in Pubmed: 30928584.

17. Szmuda T, Słoniewski P, Sabisz A, et al. Traktografia nerwu twarzowego w guzach kąta mostowo-móżdżkowego. Otorynolaryngologia. 2015; 13: 42-50.

18. Roundy N, Delashaw JB, Cetas JS. Preoperative identification of the facial nerve in patients with large cerebellopontine angle tumors using high-density diffusion tensor imaging. J Neurosurg. 2012; 116(4): 697-702, doi: 10.3171/2011.12.JNS111404, indexed in Pubmed: 22283188.

19. Choi KS, Kim MS, Kwon HG, et al. Preoperative identification of facial nerve in vestibular schwannomas surgery using diffusion tensor tractography. J Korean Neurosurg Soc. 2014; 56(1): 11-15, doi: 10.3340/jkns.2014.56.1.11, indexed in Pubmed: 25289119.

20. Zhang Y, Chen Y, Zou Y, et al. Facial nerve preservation with preoperative identification and intraoperative monitoring in large vestibular schwannoma surgery. Acta Neurochir (Wien). 2013; 155(10): 1857-1862, doi: 10.1007/s00701-013-1815-9, indexed in Pubmed: 23877233.

21. Yoshino M, Kin T, Ito A, et al. Feasibility of diffusion tensor tractography for preoperative prediction of the location of the facial and vestibulocochlear nerves in relation to vestibular schwannoma. Acta Neuro- chir (Wien). 2015; 157(6): 939-46; discussion 946, doi: 10.1007/ s00701-015-2411-y, indexed in Pubmed: 25862170.

22. Wei PH, Qi ZG, Chen Ge, et al. Identification of cranial nerves near large vestibular schwannomas using superselective diffusion tensor tractography: experience with 23 cases. Acta Neurochir (Wien). 2015; 157(7): 1239-1249, doi: 10.1007/s00701-015-2431-7, indexed in Pubmed: 25948078.

23. Gerganov VM, Giordano M, Samii M, et al. Diffusion tensor imaging -based fiber tracking for prediction of the position of the facial nerve in relation to large vestibular schwannomas. J Neurosurg. 2011; 115(6): 1087-1093, doi: 10.3171/2011.7.JNS11495, indexed in Pubmed: 21962081.

24. Jacquesson T, Cotton F, Frindel C. MRI Tractography Detecting Cranial Nerve Displacement in a Cystic Skull Base Tumor. World Neurosurg. 2018; 117: 363-365, doi: 10.1016/j.wneu.2018.06.182, indexed in Pubmed: 29966790.

25. Yoshino M, Kin T, Ito A, et al. Diffusion tensor tractography of normal facial and vestibulocochlear nerves. Int J Comput Assist Radiol Surg. 2015; 10(4): 383-392, doi: 10.1007/s11548-014-1129-2, indexed in Pubmed: 25408307.

26. Zolal A, Juratli TA, Podlesek D, et al. Probabilistic Tractography of the Cranial Nerves in Vestibular Schwannoma. World Neurosurg. 2017; 107: 47-53, doi: 10.1016/j.wneu.2017.07.102, indexed in Pubmed: 28754643.

27. Yoshino M, Kin T, Ito A, et al. Combined use of diffusion tensor tractography and multifused contrast-enhanced FIESTA for predicting facial and cochlear nerve positions in relation to vestibular schwannoma. J Neurosurg. 2015; 123(6): 1480-1488, doi: 10.3171/2014.11. JNS14988, indexed in Pubmed: 26053235.

28. Pereira P, Manacas R. Magnetic Resonance Imaging Fusion of Cranial Nerves Impaired by Skull Base Tumors: A Technical Development. Journal of Nuclear Medicine \& Radiation Therapy. 2019; 10(1), doi: 10.4172/2155-9619.1000396.

29. Breun M, Nickl R, Perez J, et al. Vestibular Schwannoma Resection in a Consecutive Series of 502 Cases via the Retrosigmoid Approach: Technical Aspects, Complications, and Functional Outcome. World Neurosurg. 2019; 129: e114-e127, doi: 10.1016/j. wneu.2019.05.056, indexed in Pubmed: 31100515. 